\title{
Preclinical Evaluation of the Pan-FGFR Inhibitor LY2874455 in FRS2-Amplified Liposarcoma
}

\author{
Robert Hanes 1,2®, Else Munthe ${ }^{1}$, Iwona Grad ${ }^{1}$, Jianhua Han ${ }^{3}$, Ida Karlsen ${ }^{3,4}$, \\ Emmet McCormack ${ }^{3,5}$, Leonardo A. Meza-Zepeda ${ }^{1,2,6}$, Eva Wessel Stratford ${ }^{1}$ \\ and Ola Myklebost 1,2,7,*(D) \\ 1 Department of Tumor Biology, Institute of Cancer Research, the Norwegian Radium Hospital, \\ Oslo University Hospital, 0379 Oslo, Norway; Robert.Hanes@rr-research.no (R.H.); \\ Else.Munthe@rr-research.no (E.M.); Iwona.Grad@rr-research.no (I.G.); \\ Leonardo.A.Meza-Zepeda@rr-research.no (L.A.M.-Z.); eva.wessel.stratford@rr-research.no (E.W.S.) \\ 2 Norwegian Cancer Genomics Consortium, 0379 Oslo, Norway \\ 3 Centre for Cancer Biomarkers (CCBIO), Department of Clinical Sciences, University of Bergen, 5021 Bergen, \\ Norway; Jianhua.Han@uib.no (J.H.); idakarlsenemail@gmail.com (I.K.); \\ emmet.mc.cormack@med.uib.no (E.M.) \\ 4 KinN Therapeutics AS, 5021 Bergen, Norway \\ 5 Department of Internal Medicine, Hematology Section, Haukeland University Hospital, \\ 5021 Bergen, Norway \\ 6 Genomics Core Facility, Department of Core Facilities, Institute of Cancer Research, the Norwegian Radium \\ Hospital, Oslo University Hospital, 0379 Oslo, Norway \\ 7 Department of Clinical Science, University of Bergen, 5020 Bergen, Norway \\ * Correspondence: ola.myklebost@uib.no; Tel.: +47-5558-4565
}

Received: 16 January 2019; Accepted: 18 February 2019; Published: 21 February 2019

\begin{abstract}
Background: FGFR inhibition has been proposed as treatment for dedifferentiated liposarcoma (DDLPS) with amplified FRS2, but we previously only demonstrated transient cytostatic effects when treating FRS2-amplified DDLPS cells with NVP-BGJ398. Methods: Effects of the more potent FGFR inhibitor LY2874455 were investigated in three DDLPS cell lines by measuring effects on cell growth and apoptosis in vitro and also testing efficacy in vivo. Genome, transcriptome and protein analyses were performed to characterize the signaling components in the FGFR pathway. Results: LY2874455 induced a stronger, longer-lasting growth inhibitory effect and moderate level of apoptosis for two cell lines. The third cell line, did not respond to FGFR inhibition, suggesting that FRS2 amplification alone is not sufficient to predict response. Importantly, efficacy of LY2874455 was confirmed in vivo, using an independent FRS2-amplified DDLPS xenograft model. Expression of FRS2 was similar in the responding and non-responding cell lines and we could not find any major difference in downstream FGFR signaling. The only FGF expressed by unstimulated non-responding cells was the intracellular ligand FGF11, whereas the responding cell lines expressed extracellular ligand FGF2. Conclusion: Our study supports LY2874455 as a better therapy than NVP-BGJ398 for FRS2-amplified liposarcoma, and a clinical trial is warranted.
\end{abstract}

Keywords: FRS2; FGFR; NVP-BGJ398; LY2874455; sarcoma

\section{Introduction}

Sarcomas are rare cancers of mesenchymal origin, accounting for approximately $1 \%$ of all solid cancers, and can be classified into more than 50 distinct histological subtypes [1]. Liposarcomas (LPS), which resemble adipose tissue, are further classified into three main subtypes, well-differentiated/dedifferentiated liposarcoma (WD/DDLPS), myxoid/round cell liposarcoma, 
and pleomorphic liposarcoma [2]. The heterogeneity makes clinical research and trials challenging. However, all together rare cancers comprise one of the largest patient groups, which is in great need of new therapeutic approaches. Therefore, a deeper mechanistic understanding is needed to be able to identify and validate new potential targets. In a previous study, we identified amplifications of multiple genes in the 12q14.1-q15 region in the DDLPS cell line NRH-LS1 and investigated several of these as therapeutic targets [3]. One of these, FRS2, is generally co-amplified with MDM2 in WD/DDLPS [4]. FRS2 codes for an important component of the FGF receptor (FGFR) signaling pathway, which plays crucial roles in multiple biological processes, such as cell growth, survival and differentiation, as well as tumor development and progression [5,6]. FRS2-dependent FGFR signaling is induced through FGFR activation by FGF ligands, and consecutive phosphorylation of FRS2 triggers an intracellular signaling cascade involving RAS/MAPK/ERK and PI3K/AKT [7], leading to oncogenic pro-survival and anti-apoptotic properties and increased proliferation and migration. To date, there are no drugs available that can target FRS2 directly, but attenuating the signal from FGFR, upstream of FRS2, with FGFR inhibitors has been shown to be growth inhibitory in such cells [3,8].

NVP-BGJ398, which is in phase II clinical trials, has been shown to be a potent and selective FGFR inhibitor in a wide panel of cancer cell lines [9]. NVP-BGJ398 has been reported to selectively inhibit FGFR1, -2 and -3 with IC50s of $0.9 \mathrm{nM}, 1.4 \mathrm{nM}$ and $1.0 \mathrm{nM}$, respectively, whereas the IC50 for FGFR4 is $60 \mathrm{nM}$ [10]. Another pan-FGFR inhibitor, LY2874455, recently completed a phase I clinical trial [11], and has been reported to selectively inhibit FGFR1, $-2,-3$, and -4 with IC50s of $2.8 \mathrm{nM}, 2.6 \mathrm{nM}, 6.4 \mathrm{nM}$ and $6 \mathrm{nM}$, respectively [12].

In this study, we have investigated the therapeutic potential of LY2874455 with the aim to improve efficacy for FRS2-amplified DDLPS.

\section{Materials and Methods}

\subsection{Cell Line and Culture Conditions}

The DDLPS cell lines NRH-LS1, established from a patient-derived xenograft as previously described [3] and LPS510 and LPS853, kindly provided by Dr. Jonathan Fletcher, were cultured in RPMI-1640 medium (Sigma-Aldrich, St. Louis, MO, USA) supplemented with 10\% FBS (Sigma-Aldrich), 1\% L-alanyl-L-glutamine (Sigma-Aldrich) and 1\% penicillin-streptomycin (Sigma-Aldrich) and grown at $37^{\circ} \mathrm{C}, 5 \% \mathrm{CO}_{2}$. Short tandem repeat DNA profiling was performed on all cell lines to confirm identity. Cells were negative for mycoplasma using the VenorGeM Mycoplasma Detection Kit (Minerva Biolabs, Berlin, Germany).

\subsection{Drugs}

LY2874455 (\#S7057) (Selleck Chemicals, Munich, Germany) was dissolved in DMSO (Sigma-Aldrich) according to the manufacturer's recommendation. For each experiment the appropriate control (referred to as untreated) was used, with a DMSO concentration corresponding to that used with the highest drug concentration. The concentration of DMSO in the control for $100 \mathrm{nM}$ LY2874455 and NVP-BGJ398 was $0.01 \%$ and for $1 \mu \mathrm{M}$ LY28744555 and NVP-BGJ398 0.1\%.

\subsection{Drug Treatment and Cell Proliferation Assay}

The cellular proliferation rate was measured using a live-cell imaging system, IncuCyte ZOOM (Essen Bioscience, Birmingham, UK) with the corresponding software application (version 2013BRev1), (Essen Bioscience, Birmingham, UK). Cells were seeded into 96-well plates, and drug treatment initiated after 16h, in triplicates. Each drug treatment was performed over a period of 1-2 weeks and done at least three times. Proliferation rate was measured as cell confluence over time every third hour. 


\subsection{Apoptosis}

Apoptosis assays were done with the IncuCyte ZOOM. To measure apoptosis, the CellPlayer 96-Well Kinetic Caspase-3/7 reagent containing DEVD-NucViewTM488 (Essen Bioscience) at a concentration of $2 \mu \mathrm{M}$ was added at the same time as the drug. Total numbers of apoptotic cells were counted in the green channel $(488 \mathrm{~nm})$. After $96 \mathrm{~h}$, cells were incubated for 30 minutes with $4 \mu \mathrm{M}$ of Nuclear-ID Red DNA stain (Enzo Life Sciences, Farmingdale, NY, USA), and total cell count was measured in the red channel $(566 \mathrm{~nm})$. The percentage of apoptotic cells per well was calculated as the number of apoptotic cells relative to the total number of nuclei.

\subsection{Viability Assay for Dose-Response Curve and IC50 Calculations}

Cell viability was measured using the CellTiter-Glo Luminescent Cell Viability Assay (Promega, Madison, WI, USA). $5 \times 10^{3}$ cells per well were seeded onto a 96-well flat and clear bottom polystyrene treated microplate (Corning, Corning, NY, USA). The drug treatment was initiated $16 \mathrm{~h}$ after seeding and was applied at concentrations ranging from $0.1 \mathrm{nM}$ to $1000 \mathrm{nM}$. After 72,96 and $120 \mathrm{~h}$, ATP levels were used as a measure of viability. Relative IC50 for $120 \mathrm{~h}$ was calculated using a four-parameter logistic function [13] based on non-linear regression analysis using SigmaPlot (Systat Software Inc, San Jose, CA, USA) version 12.5.0.38.

\subsection{In Vivo Assay}

Animal experiments were performed according to protocols approved by the National Animal Research Authority (Mattilsynet) in compliance with the European Convention of the Protection of Vertebrates Used for Scientific Purposes (ID 10175). The LS70x xenograft (established directly from a DDLPS tumor) was implanted into the flank of immunodeficient NOD-scid IL2r $\gamma$ null (NSG) mice [14]. When tumors reached $150 \mathrm{~mm}^{3}$, animals were randomized into two groups, each of six mice, and treated twice per day with either $3 \mathrm{mg} / \mathrm{kg}$ LY2874455 or vehicle only ( $2 \%(v / v)$ DMSO, $30 \%(v / v)$ PEG 300, 5\% $(v / v)$ Tween 80 in sterile water), administered by oral gavage. Treatment was performed for 28 days, or until the tumor reached a size of $1 \mathrm{~cm}^{3}$. Tumor growth was measured by caliper measurements twice per week for the duration of treatment. Unpaired two-tailed $t$-test was performed to detect significant differences in tumor volumes ( $p \leq 0.05$ was considered significant).

\subsection{Western Blots}

Cells were treated for $24 \mathrm{~h}$ with either $100 \mathrm{nM}$ LY2874455, $100 \mathrm{nM}$ NVP-BGJ398 or control-treated with the corresponding concentration of DMSO and the last $15 \mathrm{~min}$ with or without $15 \mathrm{ng} / \mathrm{ml}$ of recombinant human FGF1 [15] and $10 \mathrm{U} / \mathrm{mL}$ of Heparin. In vitro cells were washed with PBS and dissolved in SDS lysis buffer. Xenografts were cut into smaller pieces and snap frozen. Proteins were extracted with T-Per lysis buffer (Thermo Fisher Scientific, Waltham, MA, USA), supplemented with protease and phosphatase inhibitors (both from Thermo Fischer Scientific), using the TissueLyser LT (QIAGEN, Venlo, Netherlands). DTT was added to the lysates before boiling. Proteins were separated in a 4-12\% Novex PAGE gel in MOPS running buffer, and transferred to PVDF membranes (Thermo Fisher Scientific). The following antibodies were used: pFRS2-TYR436 (\#3861), AKT (\#9272), pAKT-SER473 (\#9271), ERK (\#9102), pERK-T202/Y204 (\#4370), PLC $\gamma 1$ (\#5690), pPLC $\gamma 1$-TYR783 (\#2821) (all from Cell Signaling Technology, Danvers, MA, USA), FRS2 (\#SC8318) (Santa Cruz Biotechnology, Dallas, TX, USA) and $\alpha$-Tubulin (\#CP06) (Merck KGaA, Darmstadt, Germany). All antibodies were diluted 1:1000, except FRS2 (1:500) and $\alpha$-Tubulin (1:2000). Secondary antibodies were rabbit anti-mouse immunoglobulins/HRP (\#P0260) and goat anti-rabbit immunoglobulins/HRP (\#P0448) (Dako, Glostrup, Denmark) at a concentration of $1.3 \mathrm{~g} / \mathrm{L}$ and $0.25 \mathrm{~g} / \mathrm{L}$ respectively. The Western blots were developed using the Supersignal Western Dura substrate (Thermo Fisher Scientific), and detected and quantified on a Syngene G-Box (Synoptics Group, Cambridge, UK) with the GeneSnap (version 7.12, Synoptics Group) and the GeneTools (version 4.3.7.0, Synoptics Group) programs, respectively. 


\subsection{Quantitative Real-Time PCR-Based Copy Number Assay}

DNA was isolated from cells using the AllPrep DNA/RNA Mini Kit (QIAGEN) according to the manufacturer's protocol. Quantitative real-time PCR was performed based on absolute quantitation using the Applied Biosystems 7900HT fast real-time PCR system (Applied Biosystems, Foster By, CA, USA). The copy numbers of FRS2 (Hs02860563_cn), ALB (Hs05929625_cn) and LSAMP (Hs05902664_cn) were determined using TaqMan copy number assays from Applied Biosystems, ALB and LSAMP were used as endogenous controls, as these have low level of DNA copy number changes in a large panel of liposarcoma samples [16]. The copy numbers were determined using the CopyCaller Software v2.1 program (Applied Biosystems) as described by the manufacturer, and the FRS2 data were normalized to $L S A M P$. The copy numbers were validated using $A L B$ as another endogenous reference gene (data not shown).

\subsection{Quantitative Real-Time PCR Based Expression Assay}

RNA was isolated from cells using the AllPrep DNA/RNA Mini Kit (QIAGEN) according to the manufacturers protocol. cDNA was prepared using $1 \mu \mathrm{g}$ of RNA and the SuperScript VILO Master Mix (Invitrogen, Carlsbad, CA, USA). Quantitative real-time PCR was performed based on $\Delta \Delta \mathrm{Ct}$ relative quantitation using the Applied Biosystems 7900HT fast real-time PCR system (Applied Biosystems). The expression levels of FRS2 were determined using TaqMan gene expression assays (Hs00183614_m1) with human B2M (VIC ${ }^{\circledR} /$ MGB probe) (Applied Biosystems) as internal control for normalization. The relative expression levels were determined using the comparative $\Delta \Delta \mathrm{Ct}$ method as described by the manufacturer. Human Adipose Tissue Total RNA was used as reference (Clontech, Mountain View, CA, USA).

\subsection{RNA Sequencing}

RNA was isolated from the cell lines using the AllPrep DNA/RNA Mini Kit (QIAGEN). mRNA sequencing libraries were prepared using $100 \mathrm{ng}$ of total RNA and the Illumina TruSeq Stranded mRNA Library Prep kit for NeoPrep following the supplier's instructions. The libraries were sequenced on a NextSeq 500 Illumina sequencer (Illumina, San Diego, CA, USA) using a High Output v2 kit chemistry, generating $2 \times 75$ bp paired-end sequence reads. RNA-Seq reads were aligned using STAR aligner (v.2.5.0b) against the human reference genome (UCSC hg19, RefSeq and Gencode gene annotations), and FPKM estimation was generated by Cufflinks 2 using the RNA-seq alignment app at Illumina BaseSpace.

\section{Results}

\subsection{Improved Efficacy Using LY2874455}

When treating NRH-LS1 cells at equivalent concentrations, LY2874455 inhibited growth of the cells more efficiently than did NVP-BGJ398 (Figure 1A). NRH-LS1 cells exposed to $100 \mathrm{nM}$ of LY2874455 were completely growth inhibited after $72 \mathrm{~h}$ (Figure 1A), while treatment with $100 \mathrm{nM}$ NVP-BGJ398 gave only partial growth inhibition at that time point. In contrast to NVP-BGJ398, we found that LY2874455 induced apoptosis in a subpopulation of NRH-LS1 cells (Figure 1B). Furthermore, $100 \mathrm{nM}$ LY2874455 induced, on average, four times higher levels of apoptosis after $96 \mathrm{~h}$, as compared to cells treated with 100 nM NVP-BGJ398 (Figure 1C). As shown in Figure 2, LY2874455 inhibited cell growth in a dose-dependent manner (Figure 2A), with the full effect at approximately $100 \mathrm{nM}$. The IC50 for LY2874455 in NRH-LS1 cells was estimated to $7 \mathrm{nM}$ compared to $47 \mathrm{nM}$ for NVP-BGJ398 (Figure S2), based on viability after $120 \mathrm{~h}$ of treatment (Figure 2B). 
A B
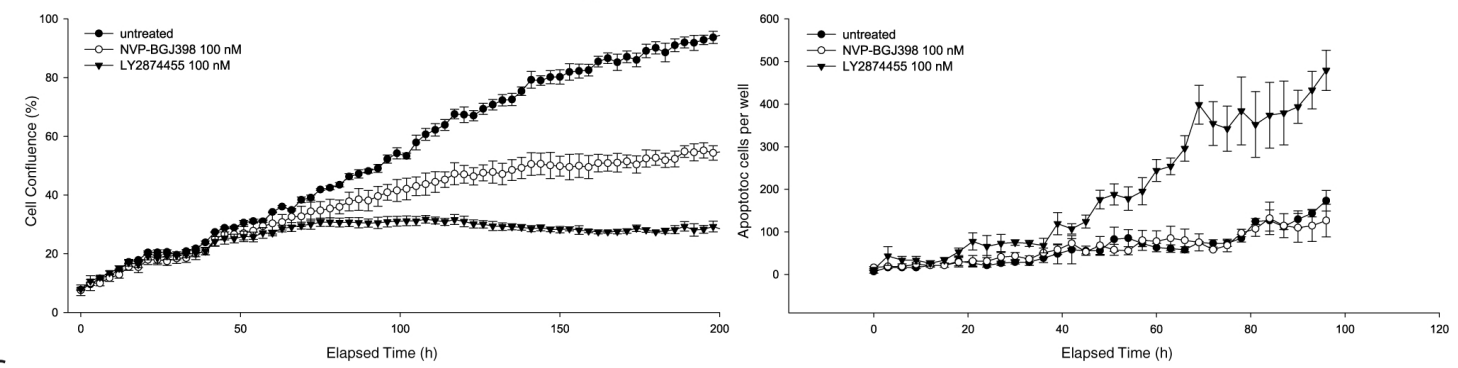

C
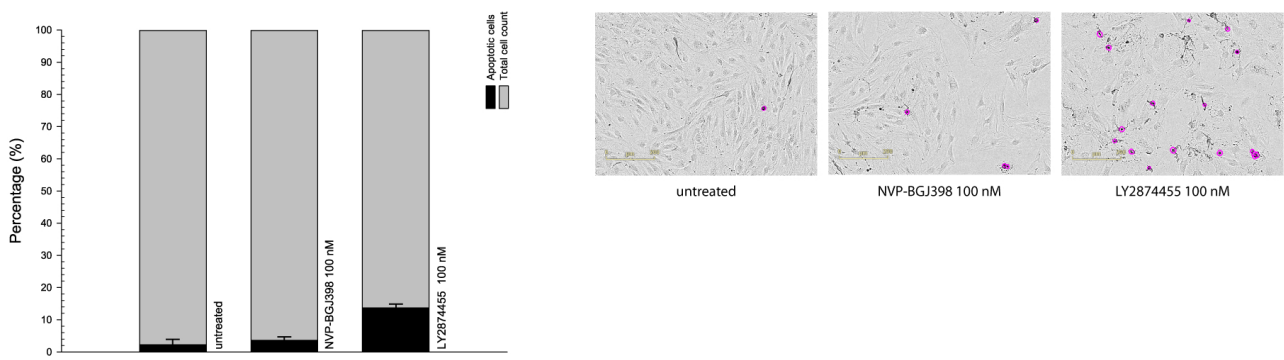

Figure 1. Comparison of the effect of NVP-BGJ398 and LY2874455 on proliferation and apoptosis of NRH-LS1 cells. (A) Proliferation of NRH-LS1 cells after inhibition of FGFR with either NVP-BGJ398 or LY2874455; one representative experiment is shown $(n=3)$, error bars represent the standard error of the mean (SEM) of technical replicates; (B) The number of cells with active caspase $3 / 7$ during $96 \mathrm{~h}$ of

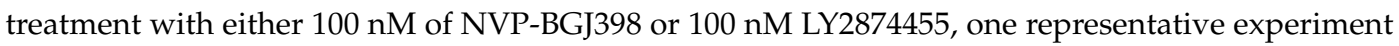
is shown $(n=3)$; (C) The percentage of apoptotic cells after $96 \mathrm{~h}$ of treatment with NVP-BGJ398 or LY2874455; the mean of experiments is shown $(n=3)$, error bars represent the standard deviation (SD) of the experiments. Representative images show apoptotic cells outlined in purple based on measured apoptotic signal. For all experiments untreated is with DMSO concentration corresponding to that of the highest drug concentration. 
A

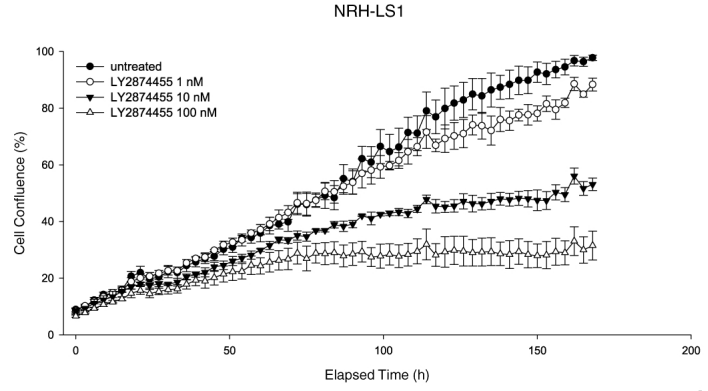

B

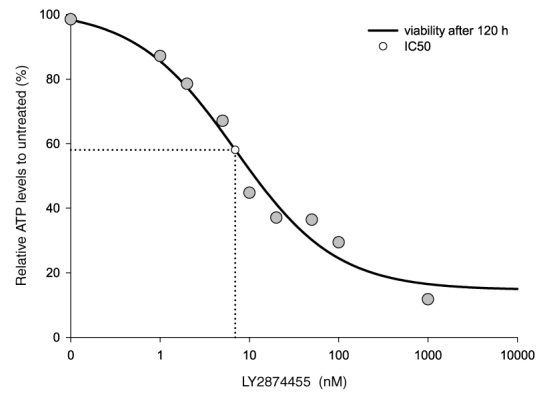

D

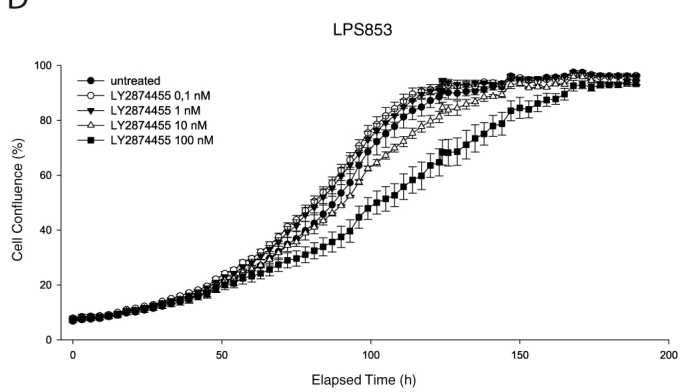

F

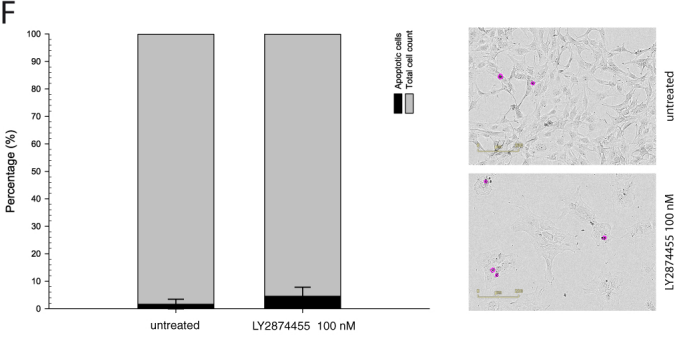

Figure 2. The effect of LY2874455 on the proliferation and viability of NRH-LS1, LPS510 and LPS853 cells. (A) Proliferation of NRH-LS1 cells at different concentrations of LY2874455; one representative experiment is shown $(n=3)$, error bars represent the standard error of the mean (SEM) of technical replicates; (B) The IC50 was estimated at $7 \mathrm{nM}$ based on NRH-LS1 cell viability after $120 \mathrm{~h}$ of treatment with LY2874455; Proliferation of LPS510 (C) and LPS853 (D) cells in the presence of LY2874455. (E) The number of LPS510 cells with active caspase $3 / 7$ during $96 \mathrm{~h}$ of treatment with $100 \mathrm{nM}$ of LY2874455; (C-E) One representative experiment is shown $(n=3)$, error bars represent the standard error of the mean (SEM) of technical replicates; (F) The percentage of apoptotic LPS510 cells after $96 \mathrm{~h}$ of treatment with $100 \mathrm{nM}$ of LY2874455. The mean of experiments is shown $(n=4)$, error bars represent the standard deviation (SD) of the experiments. Representative images show apoptotic cells outlined in purple based on measured apoptotic signal. For all experiments untreated is with DMSO concentration corresponding to that of the highest drug concentration.

\subsection{The Response to FGFR Inhibition in FRS2-Amplified Cell Lines Is Variable}

We next investigated the effect of LY2874455 in two additional FRS2-amplified DDLPS cell lines, LPS510 and LPS853, which have FRS2 copy number and gene expression levels comparable to NRH-LS1 (Figure S1). The expression levels of FRS2 were 10-20 fold higher in all three cell lines compared to both human adipocyte tissue and an undifferentiated immortalized mesenchymal progenitor cell line (iMSC\#3), [17] (Figure S1). LY28744555 inhibited the growth of LPS510 similar to NRH-LS1 (Figure 2C), with IC50 values of 5.5 and 6.9 respectively (Figure S2). In contrast, LPS853 was only modestly inhibited at $100 \mathrm{nM}$ (Figure 2D). Similar levels of apoptosis were observed for both LPS510 and NRH-LS1 after treatment with $100 \mathrm{nM}$ LY2874455, with 9\% for LPS510 cells (Figure 2E,F) and 13\% for NRH-LS1 (Figure 1C) at $96 \mathrm{~h}$. 


\subsection{LY2874455 Induces Long-Lasting Growth Inhibition}

In order to assess duration of the growth inhibitory effects of LY2874455 and NVP-BGJ398, we discontinued drug treatment after $96 \mathrm{~h}$ or $264 \mathrm{~h}$. In contrast to NVP-BGJ398, the growth inhibition of NRH-LS1 and LPS510 was strong after withdrawal of LY2874455, although it was quite significant with LPS510 cells, especially at the highest doses (Figure 3A-D). Both LPS510 and NRH-LS1 maintained growth arrest within the time-frame of the experiment after $264 \mathrm{~h}$ of treatment with LY2874455.

A

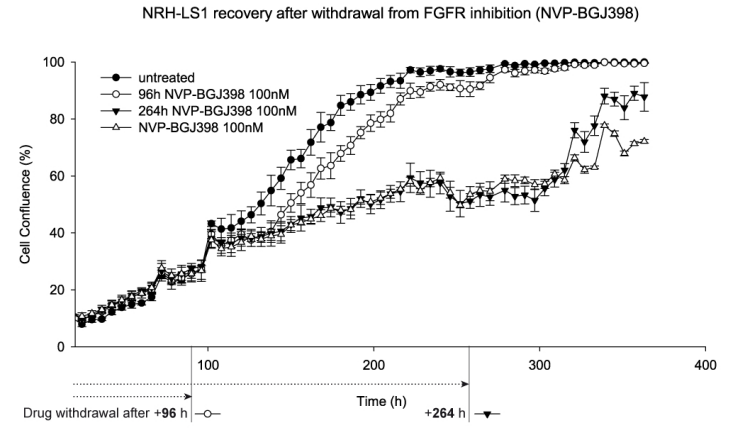

C

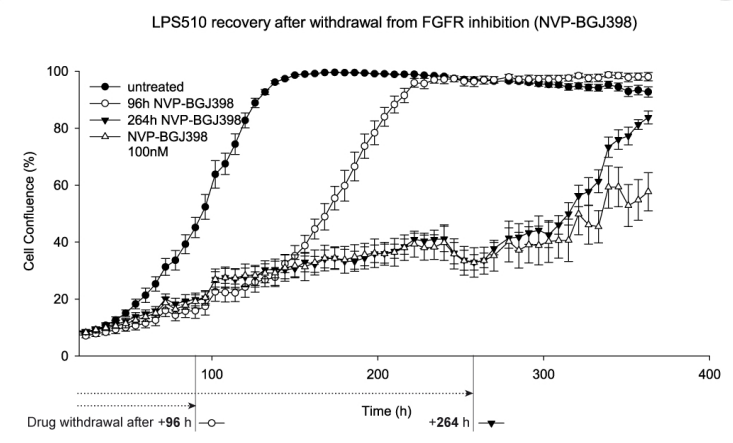

B

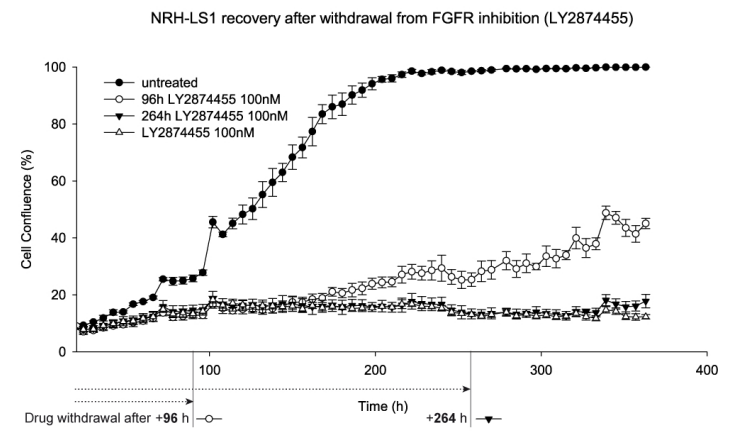

D

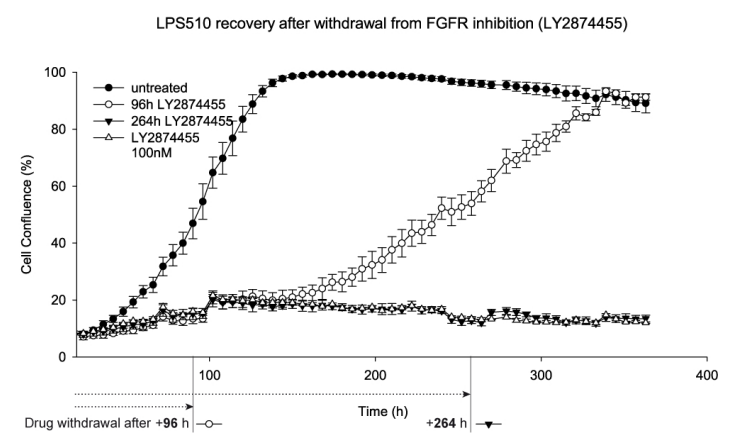

Figure 3. NRH-LS1 and LPS510 cells resume proliferation after withdrawal of treatment with NVP-BGJ398, but not LY2874455. Proliferation of NRH-LS1 (A,B) or LPS510 (C,D) cells treated with $100 \mathrm{nM}$ of NVP-BGJ398 (A,C) or LY2874455 (B,D) continuously or upon withdrawal of the drug after $96 \mathrm{~h}$ or $264 \mathrm{~h}$ of treatment. One representative experiment is shown $(n=3)$, error bars represent the standard error of the mean (SEM) of technical replicates. For all experiments untreated with DMSO concentration corresponding to that of the highest drug concentration.

\subsection{Both FGFR Inhibitors Inactivate MEK/ERK-Dependent Signaling in FRS2-Amplified Cells}

To determine whether the different effects of these drugs could be attributed to the activation or inhibition of different signaling components in the FGFR pathway, we investigated the status of several signaling proteins downstream of FGFR and FRS2 in NRH-LS1 cells upon stimulation with FGF and treatment with NVP-BGJ398 or LY2874455 (Figure 4, quantified in Figure S4A). Although phosphorylation of FRS2 was expected to drive growth in these cells, we only detected phosphorylated FRS2 upon stimulation with exogeneous FGF, probably because pFRS2 levels in unstimulated cells were below the detection limit of the pFRS2 antibody used in this western blot assay. An increased phosphorylation of the downstream signaling protein ERK was also found upon stimulation with FGF1. When NRH-LS1 cells were treated for $24 \mathrm{~h}$ with either $100 \mathrm{nM} \mathrm{LY2874455}$ or 100 nM NVP-BGJ398, FGF1-induced phosphorylation of FRS2 was completely abolished (Figure 4A), supporting the expected drug action, and also endogenous pERK was reduced. Stimulation with FGF1 also induced phosphorylation of PLC $\gamma 1$, an FRS2-independent component of the FGFR pathway, which was completely abolished by the treatment with either FGFR inhibitor. The expression and phosphorylation of AKT was unaffected by FGF1 stimulation or FGFR inhibition (Figure 4A). Thus, 
no clear differences in FGF signaling could be seen that explained the difference in growth inhibition of the two inhibitors in NRH-LS1.

A

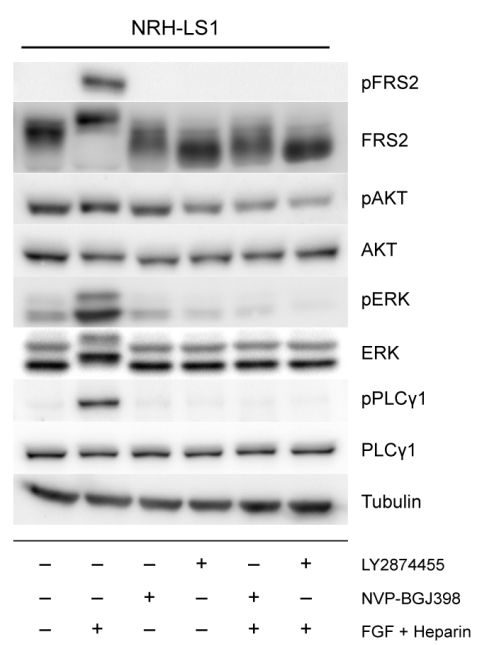

B

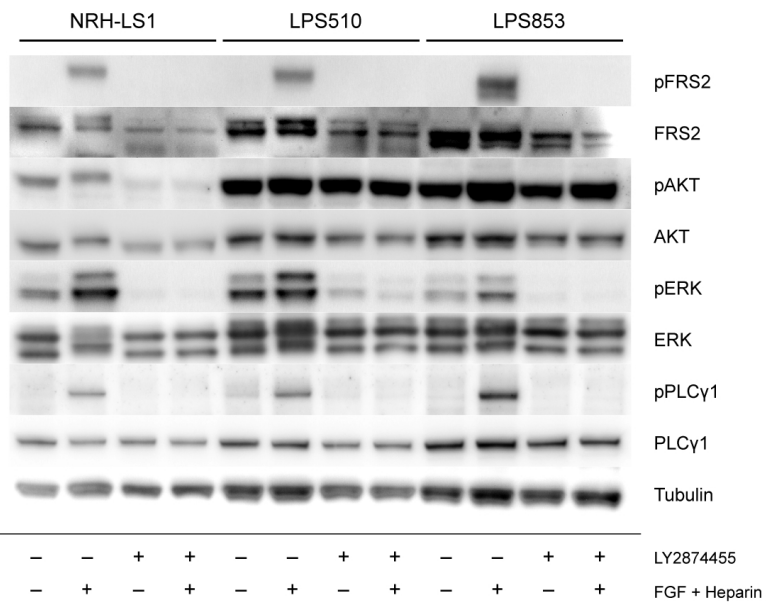

Figure 4. Signaling pathway analysis after FGFR stimulation and inhibition in NRH-LS1, LPS510 and LPS853. Western blots showing (A) The level of phosphorylated and total protein for the indicated proteins in NRH-LS1 cells treated for $24 \mathrm{~h}$ with $100 \mathrm{nM}$ of LY2874455 or $100 \mathrm{nM}$ NVP-BGJ398, with or without FGF stimulation as indicated; (B) The level of phosphorylated and total protein for the indicated proteins in NRH-LS1, LPS510 and LPS853 cells treated for $24 \mathrm{~h}$ with $100 \mathrm{nM}$ of LY2874455, with or without FGF as indicated. In all Western blot experiments $\alpha$-tubulin was used as a loading control.

We further compared the effect of LY2874455 on the same proteins on the three cell lines to understand their difference in sensitivity to FGFR inhibition (Figure 4B, quantified in Figure S4B). The western blots confirmed that FRS2 protein was expressed in all three cell lines, while pFRS2 was below detection in unstimulated cells. Phosphorylation of FRS2, PLC $\gamma 1$, and ERK was induced by stimulation with FGF1 and was inhibited upon treatment with LY2874455 in all three cell lines, showing the ability of these cell lines to respond to FGF stimulation and FGFR inhibition. The phosphorylation levels of AKT were higher in both LPS510 and LPS853 compared to NRH-LS1 but remained unaffected by stimulation or inhibition of FGFR signaling (Figure 4B).

\subsection{The Expression of FGF Receptors Varies in FRS2-Amplified LPS Lines}

In order to investigate why only two out of three LPS cell lines responded to the treatment with FGFR inhibitors and whether this could be explained due to a differential expression of some components of the FGFR pathways, we analyzed transcriptome sequencing data of NRH-LS1, LPS510, and LPS853 for expression of FGFR signaling components upstream of FRS2. Similar relative expression (FPKM) values of 52.0, 51.8 and 44.3 for FRS2 were found in NRH-LS1, LPS510 and LPS853, respectively. All three cell lines also had similar expression levels for FGFR1 with FPKMs of 41.5, 51.5 and 36.1, while FGFR4 was comparably higher expressed in LPS853 with an FPKM of 77.5, compared to 1.2 and 0 in NRH-LS1 and LPS510, respectively. All the cell lines had very low expression of FGFR2 and FGFR3. FGFRL1 is another member of the fibroblast growth factor receptor family, however it lacks the cytoplasmic tyrosine kinase domain and can act as a negative regulator of FGFR signaling [18]. Interestingly, FGFRL1 was higher expressed in LPS853 than in LPS510 and NRH-LS1, with FPKM values of 50.0, 25.8 and 11.1, respectively.

We also found FGF2 to be higher expressed in NRH-LS1 and LPS510 with FPKM values of 19.6 and 21.0 respectively, compared to LPS853 with FPKM of 0.5. In turn, FGF11 expression was higher in LPS853 with FPKM of 24.5, compared to NRH-LS1 and LPS510 with values of 0.4 and 1.2 respectively. 
A heat map that shows the expression of FGF receptors and ligands, as well as adapter proteins, is provided in Figure S3.

\subsection{LY2874455 Inhibits Tumor Growth In Vivo}

Having observed promising therapeutic potential in vitro for two out of three FRS2-amplified LPS cell lines treated with LY2874455, we investigated the effect in vivo. Patient-derived xenograft (PDX) models derived directly from patient material are more representative for drug responses, but the NRH-LS1 PDX model, from which the cell line is derived, grows slowly and was not suitable for preclinical testing. Rather than making a less representative cell line-derived PDX from LPS510, we used the DDLPS patient-derived LS70x PDX [14], which also provided an additional independent model. LS70x has amplification and increased expression of FRS2 comparable to the three cell lines (Figure S1), and grows reasonably well in NSG mice. When tumors reached $150 \mathrm{~mm}^{3}$, mice were treated with $3 \mathrm{mg} / \mathrm{kg}$ twice per day. Already from day 4 of treatment we observed significant inhibition of tumor growth compared to control-treated mice (Figure 5A). To confirm that LY2874455 reduced FGFR signaling in vivo, we performed a kinetic study of FGFR signaling proteins. Tumors were harvested at 3, 24 and $48 \mathrm{~h}$ after last treatment (end of study) and protein lysates were subjected to Western blotting to analyze the phosphorylation levels of FRS2 and ERK. The endogenous level of phosphorylated FRS2 in the LS70x tumors was, as in the cell lines, below detection (data not shown). However, ERK phosphorylation was clearly reduced $3 \mathrm{~h}$ after treatment and remained reduced for at least $24 \mathrm{~h}$ (Figure 5B, quantified in Figure S4C).

A

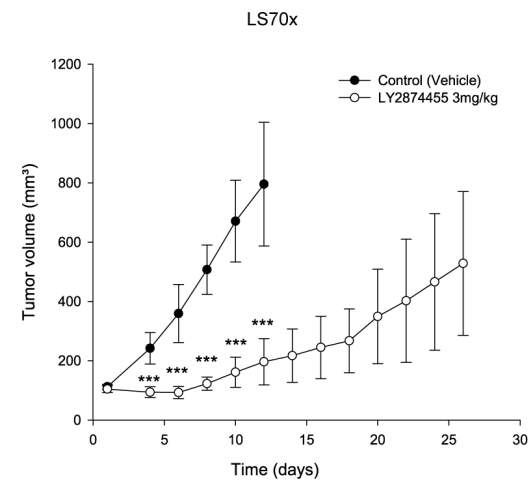

B

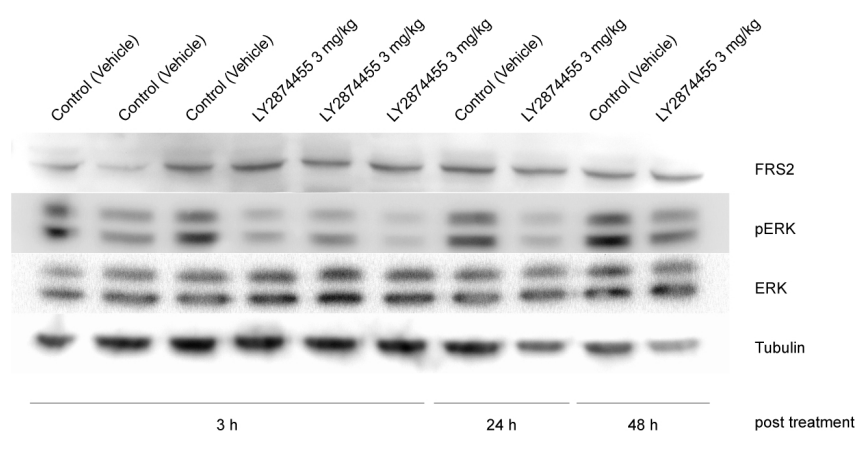

Figure 5. Growth inhibitory effect of LY2874455 in vivo on LS70x, a FRS2-amplified xenograft. (A) in vivo study with FRS2-amplified tumors of LS70x xenografts $(n=6)$ treated twice per day (BID) with LY2874455 $3 \mathrm{mg} / \mathrm{kg}$ or control vehicle for up to 28 days until tumor size reaches a limit of $1 \mathrm{~cm}^{3}$. Data shown as means $\pm \mathrm{SEM}^{* * *} p \leq 0.001$; unpaired two-tailed $t$-test treated versus vehicle treated; (B) Western blots showing the level of phosphorylated and total ERK in lysates extracted from LS70x tumors treated in vivo with vehicle or LY2874455 until endpoint. The tumors were harvested at the indicated time after last treatment. $\alpha$-tubulin is used as loading control.

\section{Discussion}

WD/DDLPS tumors almost invariably have FRS2 amplified [8] and are in great need of new therapies. We cannot expect many new drugs specific for rare cancers, therefore the possibility to repurpose existing drugs for these patients deserves thorough consideration. FGF receptor inhibitors have shown tolerable toxicities in rodent and patients $[10,11]$, thus showing efficacy in preclinical models could pave the way to clinical trials on sarcoma patients.

Our previous in vitro study demonstrated limitations of NVP-BGJ398, since the drug was only transiently cytostatic and the cells quickly regained growth capacity when drug was removed [3]. In this study we further investigated the potential of FGFR inhibition as treatment for FRS2-amplified DDLPS. 
Results were more promising using the FGFR inhibitor LY2874455, which gave improved efficacy in vitro. LY2874455 is reported to have a similar potency against all four FGFRs in biochemical assays and has shown potent activity against FGFR signaling in preclinical studies of several cancer types such as lung, gastric, bladder and multiple myeloma [12]. This drug had a stronger effect on the growth of NRH-LS1 cells, and induced apoptosis more efficiently than NVP-BGJ398 did. Interestingly, LY2874455 had a long-lasting effect on the responding cell lines, with cell growth inhibited several days after the drug treatment was discontinued. The observed effect and higher potency of LY2874455 compared to NVP-BGJ398 could potentially be due to off-target inhibition, since LY2874455 was shown to act as a multi-kinase inhibitor and consequently inhibits a wide range of different kinases [19]. However, the similarity of the response of FGFR signaling by the two inhibitors indicates a similar mode of action.

We hypothesized that amplified FRS2 would potentiate FGFR signaling and drive growth of FRS2-amplified DDLPS. Thus, FRS2 amplification could be a biomarker predicting sensitivity to FGFR inhibitors. Although the three FRS2 amplified cell lines had similar levels of FRS2, LPS853 was resistant, suggesting that FRS2 amplification alone is not sufficient as predictive biomarker. The lack of response of one cell line out of four independent models might not be representative of the patient population, but more refined biomarkers detecting such tumors would be valuable. To identify possible differences in FGFR signaling that could explain the different responses to FGFR inhibition, we analyzed the status of FGFR signaling pathway proteins. The basal level of phosphorylated FRS2 in unstimulated cells was undetectable but was drastically increased upon addition of FGF1 for all the three cell lines, confirming functional FGFR signaling. Both AKT and ERK are downstream of FRS2 and the strong inhibition of ERK phosphorylation when cells were treated with FGFR inhibitor indicated that the FGFR pathway is the predominant activator of ERK in these cells. This is consistent with a study in human bladder cancer, which showed dephosphorylation of FRS2 and ERK by NVP-BGJ398 [10]. We could not detect significant changes in levels of AKT or phosphorylated AKT, which is also consistent with previous studies [20].

We did not observe any FGFR mutations or translocations that could explain the different response to FGFR inhibition (data not shown). Although we observed equal expression of FGFR1 among the cell lines, the non-responding LPS853 cells had considerably higher expression of FGFR4. Furthermore, LPS853 also had a high expression of FGFRL1, which lacks the kinase domain and has been suggested to be a negative regulator of FGFR1 signaling [21]. Although LPS853 cells do not respond to FGF inhibitors with reduced proliferation, FGFR inhibition still prevents endogenous and FGF1-induced ERK phosphorylation in these cells. This implies that FGFR signaling is maintained in LPS853 cells, but that these cells are not dependent on phosphorylated ERK for proliferation. This is known for other cell types as well. Only a subset of KRAS mutated colon, pancreatic and lung cancer depends on MEK/ERK signaling for proliferation, although the cells responds to MEK inhibition with reduced pERK [22]. Often this is due to other rescuing mechanisms. This could explain why LPS853 cells grow independent of this pathway, and also why they respond to higher doses of LY2874455, but not to high doses of NVP-BGJ398 (data not shown) [3], since LY2874455, unlike NVP-BGJ398, has a similar potency against all four FGF receptors in biochemical assays [12,23].

We expect the levels of FGFs in fetal calf serum to be low, but the responding cell lines may produce autocrine FGFs, as was indeed indicated by the RNA-seq data. NRH-LS1 and LPS510 had high expression of FGF2, a ligand for several of the FGF receptors, while LPS853 had high expression of the intracellular FGF11. While the function of FGF11 is not fully known, FGF2 is known to be secreted by adipocytes and to stimulate proliferation upon binding to FGFR1. We hypothesize that the lack of expression of any extracellular FGF ligands in LPS853 cells indicate that they grow independently of FGFR in vitro, but we cannot exclude that they depended on exogeneous FGF in vivo and have adapted to conditions without FGF in vitro. Further functional investigations including knock-down of the different ligands and receptors might solve these issues. 
In summary, our results support LY2874455 as a better drug candidate than NVP-BGJ398 for treatment of FRS2-amplified liposarcoma. LY2874455 also showed significant efficacy in vivo, which is an important finding, since the FGF-regulatory landscape in tissues is different from cell cultures. Whether efficacy of LY2874455 can also be translated to other sarcomas with aberrations in the FGFR pathway, such as amplified, fused or mutated FGFR genes [24], needs to be investigated. We hope these studies could result in a clinical trial for DDLPS patients in great need of new treatments now that phase I clinical trials have shown tolerable toxicities [11].

Supplementary Materials: The following are available online at http:/ /www.mdpi.com/2073-4409/8/2/189/s1, Figures S1-S4.

Author Contributions: R.H.: conceptualization, drug studies, data curation and analysis, project administration, visualization, writing - original draft, review, and editing. I.G.: drug studies, data curation and analysis, writing-review and editing. E.M. (Else Munthe): protein studies, data curation and analysis, writing-review and editing. J.H.: animal studies, data curation and analysis, manuscript review. I.K.: animal studies, data curation and analysis, manuscript review. E.M. (Emmet McCormack): animal studies, data curation and analysis, manuscript review. L.A.M.-Z.: supervision, genomics, data curation and analysis, writing - review and editing. E.W.S.: supervision, design of animal studies, writing-review and editing. O.M.: conceptualization, funding acquisition, project administration, resources, supervision, writing-review and editing.

Funding: This research was funded by the REGIONAL HEALTH AUTHORITY FOR SOUTH-EASTERN NORWAY, grant number 2014047, the NORWEGIAN RESEARCH COUNCIL, grant number 221580, the NORWEGIAN CANCER SOCIETY, grant number PR-2007-0163, and by the LIDDY SHRIVER SARCOMA INITIATIVE for Translational Research in Well- and De-differentiated Liposarcoma.

Acknowledgments: We are grateful to Mona Mari Lindeberg for DNA and RNA isolation and Susanne Lorenz from the Genomics Core Facility at Oslo University Hospital for the sequencing of the cell lines. We would also like to thank Mihaela Popa for contributing to the design and planning of the in vivo experiment. This work was supported by grants from the Norwegian Research Council, The Regional Health Authority of South-Eastern Norway, and the Norwegian Cancer Society.

Conflicts of Interest: The authors declare no conflict of interest.

\section{References}

1. Burningham, Z.; Hashibe, M.; Spector, L.; Schiffman, J.D. The epidemiology of sarcoma. Clin. Sarcoma Res. 2012, 2, 14. [CrossRef] [PubMed]

2. Barretina, J.; Taylor, B.S.; Banerji, S.; Ramos, A.H.; Lagos-Quintana, M.; Decarolis, P.L.; Shah, K.; Socci, N.D.; Weir, B.A.; Ho, A.; et al. Subtype-specific genomic alterations define new targets for soft-tissue sarcoma therapy. Nat. Genet. 2010, 42, 715-721. [CrossRef] [PubMed]

3. Hanes, R.; Grad, I.; Lorenz, S.; Stratford, E.W.; Munthe, E.; Reddy, C.C.S.; Meza-Zepeda, L.A.; Myklebost, O. Preclinical evaluation of potential therapeutic targets in dedifferentiated liposarcoma. Oncotarget 2016, 7, 54583-54595. [CrossRef] [PubMed]

4. Wang, X.; Asmann, Y.W.; Erickson-Johnson, M.R.; Oliveira, J.L.; Zhang, H.; Moura, R.D.; Lazar, A.J.; Lev, D.; Bill, K.; Lloyd, R.V.; et al. High-resolution genomic mapping reveals consistent amplification of the fibroblast growth factor receptor substrate 2 gene in well-differentiated and dedifferentiated liposarcoma. Genes Chromosomes Cancer 2011, 50, 849-858. [CrossRef] [PubMed]

5. Wesche, J.; Haglund, K.; Haugsten, E.M. Fibroblast growth factors and their receptors in cancer. Biochem. J. 2011, 437, 199-213. [CrossRef] [PubMed]

6. Korc, M.; Friesel, R.E. The role of fibroblast growth factors in tumor growth. Curr. Cancer Drug Targets 2009, 9, 639-651. [CrossRef] [PubMed]

7. Eswarakumar, V.P.; Lax, I.; Schlessinger, J. Cellular signaling by fibroblast growth factor receptors. Cytokine Growth Factor Rev. 2005, 16, 139-149. [CrossRef] [PubMed]

8. Zhang, K.; Chu, K.; Wu, X.; Gao, H.; Wang, J.; Yuan, Y.-C.; Loera, S.; Ho, K.; Wang, Y.; Chow, W.; et al. Amplification of FRS2 and activation of FGFR/FRS2 signaling pathway in high-grade liposarcoma. Cancer Res. 2013, 73, 1298-1307. [CrossRef] [PubMed]

9. Guagnano, V.; Kauffmann, A.; Wöhrle, S.; Stamm, C.; Ito, M.; Barys, L.; Pornon, A.; Yao, Y.; Li, F.; Zhang, Y.; et al. FGFR genetic alterations predict for sensitivity to NVP-BGJ398, a selective pan-FGFR inhibitor. Cancer Discov. 2012, 2, 1118-1133. [CrossRef] [PubMed] 
10. Guagnano, V.; Furet, P.; Spanka, C.; Bordas, V.; Le Douget, M.; Stamm, C.; Brueggen, J.; Jensen, M.R.; Schnell, C.; Schmid, H.; et al. Discovery of 3-(2,6-dichloro-3,5-dimethoxy-phenyl)-1\{6-[4-(4-ethyl-piperazin-1-yl)-phenylamino]-pyrimidin-4-yl\}-1-methyl-urea (NVP-BGJ398), a potent and selective inhibitor of the fibroblast growth factor receptor family of receptor tyrosine kinase. J. Med. Chem. 2011, 54, 7066-7083. [CrossRef]

11. Michael, M.; Bang, Y.-J.; Park, Y.S.; Kang, Y.-K.; Kim, T.M.; Hamid, O.; Thornton, D.; Tate, S.C.; Raddad, E.; Tie, J. A Phase 1 Study of LY2874455, an Oral Selective pan-FGFR Inhibitor, in Patients with Advanced Cancer. Target Oncol. 2017, 12, 463-474. [CrossRef] [PubMed]

12. Zhao, G.; Li, W.-Y.; Chen, D.; Henry, J.R.; Li, H.-Y.; Chen, Z.; Zia-Ebrahimi, M.; Bloem, L.; Zhai, Y.; Huss, K.; et al. A novel, selective inhibitor of fibroblast growth factor receptors that shows a potent broad spectrum of antitumor activity in several tumor xenograft models. Mol. Cancer Ther. 2011, 10, 2200-2210. [CrossRef] [PubMed]

13. Sebaugh, J.L. Guidelines for accurate EC50/IC50 estimation. Pharm. Stat. 2011, 10, 128-134. [CrossRef] [PubMed]

14. Kresse, S.H.; Meza-Zepeda, L.A.; Machado, I.; Llombart-Bosch, A.; Myklebost, O. Preclinical xenograft models of human sarcoma show nonrandom loss of aberrations. Cancer 2012, 118, 558-570. [CrossRef] [PubMed]

15. Wesche, J.; Wiedlocha, A.; Falnes, P.O.; Choe, S.; Olsnes, S. Externally added aFGF mutants do not require extensive unfolding for transport to the cytosol and the nucleus in NIH/3T3 cells. Biochemistry 2000, 39, 15091-15100. [CrossRef] [PubMed]

16. Kanojia, D.; Nagata, Y.; Garg, M.; Lee, D.H.; Sato, A.; Yoshida, K.; Sato, Y.; Sanada, M.; Mayakonda, A.; Bartenhagen, C.; et al. Genomic landscape of liposarcoma. Oncotarget 2015, 6, 42429-42444. [CrossRef] [PubMed]

17. Skårn, M.; Noordhuis, P.; Wang, M.-Y.; Veuger, M.; Kresse, S.H.; Egeland, E.V.; Micci, F.; Namløs, H.M.; Håkelien, A.-M.; Olafsrud, S.M.; et al. Generation and characterization of an immortalized human mesenchymal stromal cell line. Stem Cells Dev. 2014, 23, 2377-2389. [CrossRef] [PubMed]

18. Sleeman, M.; Fraser, J.; McDonald, M.; Yuan, S.; White, D.; Grandison, P.; Kumble, K.; Watson, J.D.; Murison, J.G. Identification of a new fibroblast growth factor receptor, FGFR5. Gene 2001, 271, 171-182. [CrossRef]

19. Hagel, M.; Miduturu, C.; Sheets, M.; Rubin, N.; Weng, W.; Stransky, N.; Bifulco, N.; Kim, J.L.; Hodous, B.; Brooijmans, N.; et al. First Selective Small Molecule Inhibitor of FGFR4 for the Treatment of Hepatocellular Carcinomas with an Activated FGFR4 Signaling Pathway. Cancer Discov. 2015, 5, 424-437. [CrossRef]

20. Luo, L.Y.; Kim, E.; Cheung, H.W.; Weir, B.A.; Dunn, G.P.; Shen, R.R.; Hahn, W.C. The tyrosine kinase adaptor protein FRS2 is oncogenic and amplified in high-grade serous ovarian cancer. Mol. Cancer Res. 2014, 13, 502-509. [CrossRef]

21. Trueb, B. Biology of FGFRL1, the fifth fibroblast growth factor receptor. Cell. Mol. Life Sci. 2011, 68, 951-964. [CrossRef] [PubMed]

22. Halilovic, E.; She, Q.-B.; Ye, Q.; Pagliarini, R.; Sellers, W.R.; Solit, D.B.; Rosen, N. PIK3CA mutation uncouples tumor growth and cyclin D1 regulation from MEK/ERK and mutant KRAS signaling. Cancer Res. 2010, 70, 6804-6814. [CrossRef] [PubMed]

23. Wu, D.; Guo, M.; Philips, M.A.; Qu, L.; Jiang, L.; Li, J.; Chen, X.; Chen, Z.; Chen, L. Crystal Structure of the FGFR4/LY2874455 Complex Reveals Insights into the Pan-FGFR Selectivity of LY2874455. PLoS ONE 2016, 11, e0162491. [CrossRef]

24. Asano, N.; Yoshida, A.; Mitani, S.; Kobayashi, E.; Shiotani, B.; Komiyama, M.; Fujimoto, H.; Chuman, H.; Morioka, H.; Matsumoto, M.; et al. Frequent amplification of receptor tyrosine kinase genes in welldifferentiated/dedifferentiated liposarcoma. Oncotarget 2017, 8, 12941. [CrossRef] [PubMed]

(C) 2019 by the authors. Licensee MDPI, Basel, Switzerland. This article is an open access article distributed under the terms and conditions of the Creative Commons Attribution (CC BY) license (http://creativecommons.org/licenses/by/4.0/). 